Design: We looked for expression of FXIIIa in 23 cases of primary CNS lymphoma from immunocompetent patients using immunohistochemistry on formalin-fixed diagnostic biopsy specimens. 19 cases were large B-cell lymphoma, 2 were high-grade Burkittlike lymphoma, and there were one case each of lymphoblastic and marginal zone lymphoma. All cases were diagnosed at UCSF, and treated at our institution with chemotherapy that included high-dose methotrexate.

Results: Significant staining for FXIIIa was found in 13 of the 23 cases (57\%), and was absent from the remaining 10 cases $(43 \%)$. This staining appeared to co-localize with staining for CD68 but not S100 or GFAP, suggesting the positive cells were histiocytes. In some cases, FXIIIa staining appeared to localize to vessels in the tumor, suggesting either endothelial expression or localization of FXIII-positive histiocytes to vascular beds. No significant expression for FXIIIa was seen in normal brain tissue. Among the large cell lymphomas, only 1 of 6 patients (17\%) with FXIIIa-negative tumors had a durable response at one-year, while that rate was $37 \%$ overall.

Conclusions: Lack of FXIIIa expression by tumor histiocytes is found in a significant proportion of CNS lymphoma cases, and is associated with a poor clinical response to therapy, raising the possibility that a failure of immunosurveillance by tumor histiocytes may be involved in the pathogenesis of primary CNS lymphomas in immunocompetent individuals.

\section{Colorectal Intramucosal Perikarya of Ganglion Cells}

VW Tunru-Dinh, $M L W u$. University of California, Irvine College of Medicine, Irvine, CA.

Background: It is generally believed that perikarya of ganglion cells in the normal human colorectum are confined to plexuses that lie deep to the mucosa. Intramucosal perikarya of ganglion cells have been noted only in ganglioneuromas, neuronal intestinal dysplasia, and the appendix.

Design: We retrospectively reviewed 58 specimens from colorectal biopsies. For each specimen, the presence of intramucosal perikarya of ganglion cells, their number, location and grouping were recorded, as well as the diagnoses. Immunostains for neuron-specific enolase and cytomegalovirus were performed to attempt to confirm the presence of intramucosal perikarya.

Results: Eleven specimens (19\%) contained intramucosal perikarya. Intramucosal perikarya were located throughout the large intestine and occupied the muscularis mucosa and lamina propria. Intramucosal perikarya were seen in normal mucosa, hyperplastic polyps, adenomas, carcinoma, inflammatory bowel disease, and cytomegalovirus-associated colitis. Perikarya morphologically resembled microgranulomas when they were clustered and cytomegalovirus when they occurred singly. Although some perikarya were immunoreactive with neuron-specific enolase, perikarya were often absent from additional sections used to perform immunostains. Conclusions: We present the first study of colorectal intramucosal perikarya of ganglion cells. Our findings demonstrate that intramucosal perikarya of ganglion cells are common in normal and abnormal mucosa. Awareness of intramucosal perikarya is necessary to avoid confusion with microgranulomas or cytomegalovirus.

\section{Uveal Melanoma and Monosomy of Chromosome 3}

RJ Tuthill, M Skacel, EC Borden, RR Tubbs. Cleveland Clinic Foundation, Cleveland, $\mathrm{OH}$.

Background: Risk assessment for uveal melanoma currently uses attributes such as tumor size and histologic cell type. In the future, morphologic molecular pathology may complement or replace those attributes. Monosomy of chromosome 3 has been associated with an adverse outcome. Fluorescence in situ hibrydization (FISH) was used to evalute uveal melanomas for monosomy of chromosome 3.

Design: A tissue microarray was constructed using two or more cores of uveal melanomas from 15 patients. FISH using a directly labeled probe specific for the pericentrimeric region of chromosome 3 was performed. Tumor cell nucleli were evaluated for the presence of one or two chromosomes. Up to 50 nuclei were evaluated in each core. For monosomy to be present, at least $30 \%$ of the evaluated nuclei had to have no more than one chromosome.

Results: FISH was successful in 13 tumors. 8 tumors revealed monosomy 3.5 had two chromosomes 3. Follow up varied from one year to eight years. Three patients developed metastatic disease at one, three and six years. One is dead of disease. All of the patients developing metastatic disease had chromosome 3 monosomy. All 5 patients with two chromosomes 3 had no evidence of disease at last follow up that varied from one year to five years. 5 patients with monosomy 3 were alive without evidence of disease at zero to eight years follow up.

Conclusions: FISH for chromosome 3 monosomy may complement other attributes used in risk assessment of uveal melanoma.

1369 Differential Amplification and Expression of CDK6 between LowGrade Astrocytoma and Glioblastoma Multiforme

B Xu, J Pettay, M Skacel, RA Prayson, GH Barnett, RR Tubbs. The Cleveland Clinic Foundation, Cleveland, $\mathrm{OH}$.

Background: Astrocytomas are the most common primary brain tumors occurring in the adult central nervous system. Emerging evidence suggests that the development of glioblastoma multiforme (GBM) is a multi-step process and is result of a series of genetic alterations occurring over time. Our previous study using array-based comparative genomic hybridization (A-CGH) demonstrated marked difference in the extent of genomic gains and losses between GBM and low-grade astrocytoma (LGA). In this study, we validated the A-CGH results for cyclin-dependent kinase 6 (CDK6) copy number changes by fluorescence in situ hybridization (FISH) and examined the expression level of CDK6 protein between GBM and LGA by immunohistochemistry (IHC).
Design: A tissue micro-array (TMA) containing 31 GBM and 5 LGA was constructed and used for FISH and IHC. The CDK6 probe derived from a bacterial artificial clone (BAC) was labeled with SpectrumGreen dUTP. The CDK6 and CEP7 probes were hybridized with the TMA slides. Immunostaining on TMA was performed using monoclonal antibody specific for CDK6.

Results: 14 cases (45\%) of GBM showed increased copy number of CDK6, whereas all five cases of LGA displayed no changes. 5 cases of GBM exhibiting high-level amplification also showed strong immunostaining for CDK6 and the other 9 cases were moderately positive. Among the cases with no copy number changes for CDK6, 15 cases of GBM and 1 case of LGA displayed weak to moderate CDK6 staining. Conclusions: This study reveals the differential changes in CDK6 copy number between GBM and LGA. Overexpression of CDK6 protein resulting from either increasing the copy number or enhanced transcription/translation of CDK6 gene may play a role in the development of GBM and the possible progression of low-grade astrocytomas to GBM. In addition, our study demonstrates that array-based genomic profiling together with FISH validation and IHC can be effective tools for the identification of new molecular markers having potential clinical utility.

1370 Diagnostic Utility of Epithelial Markers in Separating Choroid Plexus Papillary Neoplasms from Metastatic Carcinomas with Papillary Architecture SX Yan, YL Liu, M Tung, JF Silverman. Allegheny General Hospital, Pittsburgh, PA. Background: Choroid plexus papillary neoplasms (CPPs) are epithelial tumors derived from the choroid plexus (CP). The diagnosis of choroid plexus papillary neoplasms is usually not difficult based on the histologic and clinical features. However, in patients with clinical history of a papillary carcinoma, separation of choroid plexus papillary neoplasm from metastatic carcinoma with papillary features is very important and can be occasionally challenging. The utility of immunohistochemical markers to separate choroid plexus papillary neoplasm from metastatic carcinoma with papillary features has not been investigated.

Design: A total of 38 cases including 11 normal choroid plexus, 8 choroid plexus papillary neoplasms and 20 metastatic carcinomas with papillary features metastatic to the central nervous system were retrieved. Epithelial markers of CAM5.2, CK7, CK20, CK5/6, Ber-EP4, EMA and B72.3 were evaluated. Immunostains were performed on an automated immunostainer with appropriate positive and negative controls. Statistical analysis was calculated with Chi-square method.

Results:

Expression of epithelial markers in choroid plexus papillary neoplasms (CPPs), normal choroid plexus $(\mathrm{CP})$ and metastatic carcinoma with papillary architecture

$\begin{array}{llllllll}\text { Markers } & \text { CAM5.2 } & \text { CK7 } & \text { CK20 } & \text { CK5/6 } & \text { Ber-EP4 } & \text { EMA } & \text { B72.3 }\end{array}$

$\begin{array}{llllllll}\text { Normal } & 64 \%(7 / 11) & 9 \%(1 / 11) & 0 \%(0 / 11) & 0 \%(0 / 11) & 0 \%(0 / 11) & 0 \%(0 / 11) & 9 \%(1 / 11)\end{array}$

Choroid

Plexus

$\begin{array}{llllllll}\mathrm{CPPs} & 100 \%(8 / 8) & 38 \%(3 / 8) & 13 \%(1 / 8) & 38 \%(3 / 8) & 0 \%(0 / 8) & 38 \%(3 / 8) & 25 \%(2 / 8)\end{array}$ $\begin{array}{llllllll}\text { Metastatic } & 100 \% & 75 \% & 20 \% & 10 \% & 80 \% & 70 \% & 70 \% \\ \text { carcinomas } & (20 / 20) & (15 / 20) & (4 / 20) & (2 / 20) & (16 / 20) & (14 / 20) & (14 / 20)\end{array}$ Conclusions: 1) Ber-EP4 and B72.3 are expressed in $80 \%$ and $70 \%$ of metastatic carcinoma with papillary features but none of the choroid plexus papillary neoplasms. Therefore, Ber-EP4 and B72.3 are useful immunohistochemical markers in separating metastatic carcinoma from CPPS.

2) CK7, which detected more metastatic carcinomas than CPPs, should be included in the panel of immunostains.

3) High molecular cytokeratin CK5/6 and CK20 are not useful in separating metastatic carcinoma from CPPs.

\section{Pathobiology}

1371 Persistent Increase of Immature Reticulocyte Fraction in Sickle Cell Anemia Treated with Hydroxyurea

$R$ Bagdasaryan, $F$ Chaves, $K$ Quillen, $M$ Gallinaro, $D X u$. Boston University School of Medicine, Boston, MA.

Background: Sickle cell anemia is an inherited disease. The deformed red blood cells obstruct the circulation causing severe pain, hemolytic anemia, and other complications. Recent studies have shown that hydroxyurea (HU) increases the concentration of $\mathrm{HbF}$ that prevents the polymerization of $\mathrm{HbS}$ and significantly improves the microcirculation, leading to decrease in the number of vaso-occlusive episodes in patients with sickle cell anemia (Steinberg et al., 2003). Since it has been noted that the Immature Reticulocyte Fraction (IRF), a ratio of immature reticulocytes to the total number of reticulocytes, is markedly increased in sickle cell anemia, we investigated the effect of HU on IRF, which serves as an important physiologic indicator of erythropoietic bone marrow response to anemia or tissue hypoxia.

Design: Thirty-two patients with sickle cell anemia at Boston Medical Center, Boston, MA were studied, including 16 treated with $\mathrm{HU}$ (mean age $=24$ years, M: F $=1.28$ ) and 16 without $\mathrm{HU}$ (mean age $=13$ years, $\mathrm{M}: \mathrm{F}=1.0$ ). Laboratory observations include IRF as well as $\mathrm{Hb}, \mathrm{RBC}, \mathrm{MCV}$, reticulocyte \%, absolute reticulocyte count (ARC) by automated hematology analyzer (Beckman Coulter), and HbF by HPLC. In all cases, peripheral blood smears were also reviewed. Statistical analysis was performed using student t test.

Results: The significant differences between HU-treated vs non-treated groups were observed in $\mathrm{HbF}(12.4 \%$ vs $8.0 \%$; $\mathrm{p}<0.05)$, MCV (102.7 fL vs $92.2 \mathrm{fL} ; \mathrm{p}<0.01)$, reticulocyte \% ( 8.1 vs $12.8 ; \mathrm{p}<0.01)$ and $\mathrm{ARC}(21800$ vs $35000 ; \mathrm{p}<0.01)$. Even more dramatic differences in $\mathrm{HbF}(18.8 \%)$, reticulocyte \% (5.8) and ARC (13600) were noted in the treated group with MCV greater than $100 \mathrm{fL}$ (mean 116.5; N=7). In addition, there was a marked reduction in the number of sickle cells per HPF in the 
peripheral blood smear in the higher MCV subgroup. No differences in $\mathrm{Hb}(9.4 \mathrm{~g} / \mathrm{dL}$ vs $8.6 \mathrm{~g} / \mathrm{dL} ; \mathrm{p}=0.1)$ and $\mathrm{RBC}\left(2.7 \times 10^{6} \mathrm{vs} 2.7 \times 10^{6} ; \mathrm{p}=0.3\right)$ were observed in treated group compared to non-treated group. The IRF was also similarly elevated in these two groups (0.60 vs $0.61 ; \mathrm{p}=0.4$ ) (normal: $0.16-0.36$ ).

Conclusions: Although hydroxyurea has significant effects on the RBC in sickle cell anemia including increases in $\mathrm{HbF}, \mathrm{MCV}$, and decreases in numbers of sickle cell, reticulocyte \% and ARC, the IRF remains consistently elevated in treated patients, which may imply continuous bone marrow stimulation secondary to chronic tissue hypoxia. The pathophysiologic mechanism needs to be further elucidated.

1372 Clinical Proteomics: In Vivo Molecular Signaling Profiles of Human Tumors, Pre and Post Tumor Perfusion with Experimental Chemotherapy KR Calvo, V Espina, A Rodriguez, $S$ Hoatson, EF Petricoin, HR Alexander, LA Liotta, JF Pingpank. NIH/National Cancer Institute, Bethesda, MD

Background: The future of cancer treatment lies in the development of individualized "designer" therapies tailored to the specific deranged molecular circuitry of an individual patient's tumor within the tissue microenvironment. We have generated proteomic profiles of human tumors in patients, measuring tumor and host cell response to chemotherapy in vivo, in real time along multiple protein signaling networks regulating survival, proliferation, apoptosis and differentiation.

Design: Biopsies of tumor and adjacent tissue were taken during surgery pre and immediately post perfusion with high dose chemotherapy to capture in vivo proteomic sigaling profiles. All patients were undergoing isolated hepatic perfusion (IHP) or isolated limb perfusion (ILP) for various metastatic cancers including melanoma, colonic adenocarcinoma, and adenocarcinoma with neuroendocrine features. Tissue lysates of pure populations of tumor and adjacent host cells were obtained via laser capture microdissection. Reverse phase protein microarrays were utilized to determine the activity of key proteins within multiple signaling networks including: Akt, Caspase 3, Bax, Hif1alpha, p38, cKit, EGFR, Her2, ERB2, Cox2, HSP70, NFkappaB, FAK, PKC, elF4E, STAT1, and STAT3.

Results: Proteomic profiles measuring the activity of over 18 proteins in signaling pathways were generated from small $(<1 \mathrm{~cm})$ tissue biopsies. Tumor and host cells displayed uniquely different proteomic profiles and had measurably different molecular responses to perfused chemotherapy. In all cases evaluated to date, significant changes in phosphorylation events regulating prosurvival pathways were detected in response to therapy in tumor cells relative to host cell profiles.

Conclusions: For the first time, in vivo proteomic profiles of the molecular circuitry of human tumors and host tissue have been generated with measurement of the response of the phosphoproteome to perfused therapy. This provides a unique platform for the molecular analysis of human tumors in vivo with the identification of deranged protein signaling pathways suitable for targeting by combinatorial therapy with molecular inhibitors. Analysis of the molecular response of human tumors in vivo to therapy has broad applications for the strategic design of individualized molecularly targeted therapies.

1373 Expression of Hedgehog Signaling Pathway (SHH, PTCH, SMO) in Pancreatic Ductal Carcinoma and Pancreatic Intraepithelial Neoplasia (PanIN) $Y$ Dancer, C Heard, J Albores-Saavedra. Lousiana State University Health Science Center, Shreveport, LA.

Background: The Hedgehog (Hh) signaling pathway is crucial for normal development and patterning of numerous human organs including the pancreas. Sonic hedgehog $(\mathrm{SHH})$ has recently been implicated as a crucial factor in pancreatic organogenesis and gland differentiation. Recently, dysregulation of these developmentally important genes has been implicated in cancer. In this study we analyze the expression of $\mathrm{Hh}$ signaling proteins in pancreatic ductal carcinoma.

Design: Normal pancreas, pancreatic ductal carcinoma and PanINs were examined by immunohistochemistry using antibodies against $\mathrm{Hh}$ signaling molecules: the secreted protein Sonic hedgehog (SHH), its receptor Patched (PTCH), and the PTCHassociated transmembrane protein Smoothened (SMOH). Histologic sections from 28 pancreatic ductal carcinomas were stained with antibodies against SHH, PTCH and $\mathrm{SMOH}$ (Santa Cruz, CA). Ductal lesions associated with these tumor cases were classified as PanIN-1, 2, 3, using newly defined criteria. Normal colonic tissue and pancreatic tissue from autopsy specimens were used for control. Interpretation of the results was done by three pathologists as follows: The expression of $\mathrm{SHH}$, $\mathrm{PTCH}, \mathrm{SMOH}$ was graded as positive when there was more than 5 percent of cytoplasmic staining.

Results: In our preliminary results, SHH was expressed in 17 of 28 invasive ductal carcinoma cases $(61 \%)$. Progressively higher expression of SHH was observed in ductal epithelium of PanIN-1 to -3 and in the surrounding reactive mesenchymal cells. PTCH was expressed in the epithelium of the invasive ductal carcinoma, PanIn1 to -3 and surrounding reactive mesenchymal cells in 20 of 28 cases ( $71 \%)$. However, the results of SMOH in some cases are still pending.

Conclusions: Hh signaling components: $\mathrm{SHH}$ and PTCH are strongly expressed in Pancreatic Ductal Carcinomas and PanINs. This study suggests that Hh signaling pathway may play a pivotal role in the initial development and progression of pancreatic ductal carcinoma.

1374 Distribution and Expression of Pancreatic Duodenal Transcription Factor 1 (PDX-1) in Bile Duct, Pancreas and Gastrointestinal Tract Mucosa

C Deng, YL Liu, P Cho, M Tung, JF Silverman. Allegheny General Hospital, Pittsburgh, PA.

Background: PDX-1, a pancreatic duodenal transcription factor 1, plays essential roles in regulating the development and proliferation of pancreatic exocrine and endocrine cells. Impaired transcription of PDX-1 during early pancreatic development leads to agenesis of the pancreas. In adult, PDX-1 is restricted primarily to beta cells where it regulates the expression of a number of pancreatic genes, including insulin, somatostatin, islet amyloid polypeptide, the glucose transporter type 2 and glucokinase. The distribution and expression of PDX-1 in the normal bile duct, pancreas and gastrointestinal tract mucosa have not been investigated.

Design: Total 56 cases including 8 each from benign gastro-esophageal junction, gastro-esophageal junction mucosa with intestinal metaplasia, stomach, duodenum, bile duct, pancreas and colon were selected. Immunohistochemical study for PDX-1 was performed. Sections from pancreatic islet cells were used as positive control. Results: Immunoreactivity within the nuclei was counted as positive stain. Positive PDX-1 stain was seen in the epithelial cells of gastro-esophageal junctional mucosa with or without intestinal metaplasia $(16 / 16,100 \%)$, epithelial cells of stomach $(8 / 8$, $100 \%)$, duodenum $(8 / 8,100 \%)$ and bile ducts $(8 / 8,100 \%)$. In the pancreas, the positive stain was seen in the acinic cells and islet cells $(8 / 8,100 \%)$. No immunoreactivity was detected in any of the pancreatic ductal cells. Colonic mucosa showed only luminar cytoplastic staining without nuclear reactivity.

Conclusions: PDX-1 expression was observed in the epithelial cells of gastroesophageal mucosa with or without intestinal metaplasia, gastric and duodenum epithelium, benign bile ductal cells and pancreatic acinic and islet cells. No PDX-1 immunoreactivity was detected in the benign pancreatic ductal cells. The pattern of distribution and expression of PDX-1 may be useful in elucidating the metastatic carcinoma of unknown origin.

1375 Pharmacodynamic Assessment of the Cyclin-Dependent Kinase Inhibitor Flavopiridol: Modulation of cdk Targets in Clinical Samples D Di Vizio, FP O'Connell, N Bhattacharya, GI Shapiro, M Loda. Dana Farber Cancer Institute and Brigham and Women's Hospital, Boston, MA

Background: Flavopiridol is the first potent inhibitor of cyclin-dependent kinases to enter clinical trial. In preclinical models, cell cycle arrest and apoptosis are induced in many tumor cell types. Clinical trials with flavopiridol to date have lacked pharmacodynamic assessment of inhibition of the target kinases in patients. To determine whether flavopiridol is indeed inhibiting cdks in either tumor or surrogate proliferating tissue, we have conducted pharmacodynamic studies in the context of a phase I trial in patients with advanced solid tumors using hourly infusions 3 weeks of every 4

Design: Skin biopsies $(n=12)$ or tumor sampling $(n=2)$ were performed prior to and $2 \mathrm{hrs}$ after the first infusion. Paraffin-embedded samples were analyzed by immunohistochemistry for effects of treatment on cdk targets including total and phospho-Rb (cdk2 and cdk4), total and phospho- p27 (cdk2) and p53 and cyclin D1 (cdk9). Approximately 200 nuclei were routinely scored for $0,1+$ and $2+$ staining; the sum of $1+$ and $2+$ staining was used to determine the $\%$ positive cells.

Results: Among 12 sets of paired skin biopsies, 6 demonstrated reduced staining of $\mathrm{Rb}$ [pS249/252] post treatment $(\mathrm{p}=0.025)$ and 8 demonstrated decreased $\mathrm{Rb}$ staining at the $[S 807 / 811]$ phosphorylation site $(\mathrm{p}<0.002)$. Depletion of phospho-Rb occurs while staining for total $\mathrm{Rb}$ is preserved. In addition, increases in total p27 occur post flavopiridol, consistent with decreased [pT187] phosphorylation. Consistent with inhibition of $\operatorname{cdk} 9$, the majority of paired skin biopsies demonstrated either stable or increased p53 levels post-treatment. Reduced staining of cyclin D1 was also demonstrated. In the two patients with accessible tumor tissue, staining demonstrated decreased phospho-p27, increased total p27 and decreased phospho-Rb [pS807/ 811 ] post treatment.

Conclusions: These data demonstrate that flavopiridol can induce biologic effects in tumor and surrogate proliferating tissue.

\section{Molecular and Morphological Correlates in Lung Cancer}

ME Edgerton, LJ Frey, DH Fisher. Vanderbilt University Medical Center, Nashville, TN.

Background: Current therapeutic options for patients with non-small cell lung carcinoma are based upon histological subtype, grade, and stage of the tumor. Analysis of histopathological morphologies by pathologists is the gold standard for diagnosis, grade, and pathological staging of tumors. Gene expression profiles that predict morphology can have causal relevance for the patient's outcome.

Design: Published cDNA gene expression array data for 34 non-small cell lung carcinomas was used for this data mining experiment. The cases were distributed as 15 squamous cell carcinomas (SCC), 11 adenocarcinomas (AC), and 8 large cell undifferentiated (LG) carcinomas. The grade distribution was 12 moderately (6 AC and 6 SCC), 14 (5 AC and 9 SCC) poorly, and 8 undifferentiated. Error rates were estimated using leave one out cross validation. The algorithm C5.0 was used to generate decision trees to predict differentiation (grade) from expression data in combination with histologic subtype, pathologic $\mathrm{T}$ stage, and pathologic $\mathrm{N}$ stage. The error rate, coverage, consistency, and complexity of the decision trees were used to assess their adequacy.

Results: The trees consistently bifurcated at the first node based upon the histologic subtype. Squamous cell carcinomas were discovered to be a subpopulation for whom a molecular profile showed high accuracy and coverage in predicting differentiation. Given this result, the squamous cell carcinomas were analyzed alone. These were 15 cases of 6 moderately and 9 poorly differentiated tumors. The most consistent model that emerged from the analyses used low expression of glutathione S-transferase M1 (GSTM1) to predict poor differentiation with $>90 \%$ accuracy. GSTM1 functions to conjugate hydrophobic electrophiles that can otherwise cause DNA damage. Published reports show that polymorphisms leading to low activity of GSTM1 are associated with a higher risk of developing squamous cell cancers of the head and neck, of developing squamous cell carcinoma of the lung, and with poor survival in non-small cell lung cancer. 
Conclusions: 1.) Low expression of GSTM1 predicts poor differentiation in lung SCC in this data. 2.) C5 can discover subpopulations with specific, predictive molecular profiles in a large, heterogeneous population. 3. )This approach may be useful in the development of molecular targets for therapy in cancer patients.

\section{Similar Age-Specific Incidence Rate Patterns Imply a Biological Relationship for Ovarian Cancers}

DE Henson, A Tilara, WA Anderson, A Schwartz. George Washington University Medical Center, Washington, DC; National Cancer Institute, Bethesda, MD.

Background: Our current pathologic classification of cancer is based on morphology, which only reflects one aspect of comparative tumor biology among neoplasms. We have used population-based age specific incidence rates, as a marker of tumor development and biology, to analyze graphically patterns among specific types of ovarian tumors.

Design: Data included 50,390 cases of ovarian cancer reported to the Surveillance, Epidemiology, and End Results Program of the National Cancer Institute. All cases were diagnosed between 1973 and 2001. The analysis included a comparison of the age-specific incidence rates of the different morphological types plotted on a log-log scale at 5-year intervals.

Results: Rates for endometroid carcinomas and stromal cell tumors increased until age 50 , and then graphically plateau, suggesting hormonal control. The rates of serous and mucinous tumors increased until age 60, and then plateau. Granulosa cell tumors were most common around age 45. Mullerian mixed tumors and undifferentiated carcinomas occurred predominately after age 50 . The rate patterns of serous papillary, serous surface papillary, and serous non-papillary carcinomas were similar. Rates for adenocarcinomas NOS and malignant epithelial tumors NOS increased steadily until age 85 . The agespecific rate patterns of borderline tumors compared to corresponding invasive carcinomas were also determined.

Conclusions: Age specific incidence rate patterns reflect cancer development and represent a component of tumor biology among ovarian tumors. The pattern similarity among serous tumors suggests that they represent a single biologic population and should not be divided into subtypes. Age specific rate patterns may serve as an adjunct for the biological classification of cancer.

1378 TAG-72 and MUC Protein Expression in Normal, Malignant, and Metastatic Colon Cancer

CL Hitchcock, D Gonda, LE Hitchcock, P Wen, EW Martin, Jr. The Ohio State University, Columbus, $\mathrm{OH}$.

Background: Previous studies with monoclonal antibodies to the high molecular weight TAG-72 antigen have identified the s-Tn antigenic epitope in association with intracellular and extracellular mucin in colorectal carcinomas and in regional and distant lymph nodes. Injection of I125-labeled antibodies to TAG-72 and subsequent removal of all radioactivity has resulted in significant improvement in patient survival regardless of stage. This study was undertaken to better understand the relationship of the TAG-72 associated molecules with $M U C$ gene product expression colorectal carcinoma. Design: Slides were reviewed and appropriate blocks obtained from 27 stage III colorectal carcinomas. Sections selected for study included: distant margin of resection, tumor-normal colon interface, and lymph nodes with and without metastatic disease. Monoclonal antibodies include MUC-1 and MUC-6 (Novocastra Laboratories), MUC2, MUC-4, and MUC-5AC (Zymed Laboratories), and B72.3 (Signet Laboratories) Optimal antigen specific retrival methods and immunohistochemical staining conditions were established for each antibody using Dako Autostainer.

Results: There is apparent up-regulation of Tag-72 antigen expression in colonic epithelial cells in close approximation with the tumor (20/24) as compared to similar cells at the margin (3/35). There is no similar alteration in the expression of MUC-1 (0/25 vs. $0 / 24$ ) MUC- 2 (25/25 vs. $24 / 24)$, MUC-4 ( $25 / 25$ vs. $22 / 24)$, MUC-5AC (0/25 vs. $2 / 24)$ and MUC-6 (0/25 vs. $1 / 24)$ in these two cell populations. Tumor staining was variable with each antibody. Discordant antigen expression was noted between the primary tumor and the metastatic focus; B72.3 (7/24), MUC-1 (5/25), MUC-2 (5/23), MUC-4 (2/24). No staining was noted in the lymph nodes draining the tumors. Conclusions: Neoplastic transformation in the colon alters the expression of MUC gene products and mucins in normal colonic epithelial cells. There is extensive heterogeneity in the expression of TAG-72 and MUC gene products within normal colonic mucosa, primary and metastatic tumors. TAG-72 and MUC-4 gene product exhibited similar staining patterns in primary and metastatic tumor.

Tumor Staining

$\begin{array}{lll}\text { Antibody } & \text { Tumor Staining } & \text { Staining Pattern } \\ \text { B72.3 } & 21 / 27(5 \text { focal }) & \text { Extracellular \& cellular } \\ \text { MUC-1 } & 8 / 27(6 \text { focal }) & \text { Extracellular \& cellular } \\ \text { MUC-2 } & 21 / 27(8 \text { focal }) & \text { Cellular } \\ \text { MUC-4 } & 18 / 27(4 \text { focal }) & \text { Extracellular \& cellular } \\ \text { MUC-5AC } & 2 / 27(2 \text { focal }) & \text { Weak Extracellular } \\ \text { MUC-6 } & 4 / 27(3 \text { focal }) & \text { Weak extracellular }\end{array}$

1379 Comprehensive Analysis of the Expression of the MetastasisAssociated Gene 1 (MTA1) in Neoplastic Tissue

MD Hofer, C Tapia, T-J Browne, M Mirlacher, G Sauter, MA Rubin. Brigham and Women's Hospital, Boston; Harvard Medical School, Boston; University of Basel, Basel, Switzerland; Dana-Farber Cancer Institute, Boston.

Background: The metastasis-associated gene 1 (MTA1) is over expressed in several human cancers and recent reports suggest that MTA1 may play a role in cancer progression either through transcription repression and/or hormone receptor interactions.

Design: In order to perform a broad survey of MTA1 expression in human tissues, we used a combination of in silico analysis of publicly available expression array data and high-density tissue micorarrays (TMA). Over 90 expression array studies were queried using Oncomine (www.oncomine.org), an internet-based compendium of expression array data. TMAs composed of over 3500 samples representing 130 different localized neoplasms including benign tumors as well as epithelial and hematopoietic malignancies were used to confirm protein expression.

Results: A comparison of both the expression array data and the results of immunohistochemical analysis of MTA on these TMAs confirm that MTA1 is ubiquitously expressed both in benign and malignant tumors. MTA1 expression was found to be associated with an invasive and migratory phenotype. In 7/16 expression array studies, the tissue types compared to each other were also represented on our TMAs and in all cases both approaches showed the same results. At the protein level, the highest levels of MTA1 expression were observed in diffuse B-cell lymphoma (mean staining intensity 3.9 on a scale of 1 to 4 ), basal cell carcinomas (3.7/4), and consistently in tumors with neuroendocrine differentiation such as paraganglioma (3.7/4), small cell carcinoma of the urinary bladder (3.5/4) and carcinoid tumor (3.1/ 4).

Conclusions: This study characterizes MTA1 expression for the first time across a broad spectrum of primary tumors, demonstrating expression in both benign and malignant neoplasm in addition to showing an association with tumors demonstrating an invasive phenotype and neuroendocrine differentiation. This study further supports the observation that MTA1 expression is associated with tissue invasion but may not be sufficient for the progression to metastatic stages.

\section{Evaluation of Microvessel Including Lymphatic Vessel Density} in Endometrial Carcinoma, Carcinosarcoma and Soft Tissue Sarcoma W Huang, G Liu, Y Fu. Beth Israel Deaconess Medical Center and Harvard Medical School, Boston, MA.

Background: The role of lymphatics and the presence of functional lymphangiogenesis during tumor growth and metastasis is largely unknown and under debate. It is known that carcinomas typically metastasize first to local lymph nodes through lymphatics then to distant site of the body, while the majority of soft tissue sarcomas $(>90 \%)$ notoriously metastasize early to distant sites via hematogenous route. Therefore, it is of great interest to compare microvessel especially lymphatic vessel density between carcinomas and sarcomas. To achieve this, we used D2-40, a novel monoclonal antibody that has shown to be specific for lymphatic endothelium, and CD31 which binds to all vascular endothelium, to immunostain a series of human tumors including endometrial carcinoma, carcinosarcoma and soft tissue sarcomas and evaluated intratumor microvessel density.

Design: Paraffin sections of surgical specimens from 11 patients with endometrial carcinoma, 6 patients with endometrial carcinosarcoma and 5 patients with various high-grade spindle cell sarcomas were immunostained with mouse anti-human D240, and CD 31 antibodies and the slides were assessed using an Olympus microscope. Five random images were taken from each case. Intratumor microvessel densities (MVD) were evaluated using IPLab Scientific imaging software and analysed using Kruskal-Wallis test.

Results: All tumor samples studied have intratumor CD31 positive microvessels. On average, sarcomas have the highest number of microvessels (MVD $=1.355 ; \mathrm{p}=0.027$ ) compared to endometrial carcinoma $(\mathrm{MVD}=0.517)$ and carcinosarcoma $(\mathrm{MVD}=1.065)$. The distribution of microvessels is diffuse in sarcomas but may be clustered in some carcinomas. Four out of five sarcomas did not have any intratumor D2-40 positive lymphatics, while all carcinomas had variable amounts of clustered lymphatics (MVD=0.225). In carcinosarcomas, the lymphatics were seen mostly within carcinomatous, instead of sarcomatous areas $(\mathrm{MVD}=0.110)$. In one patient who had simultaneous carcinosarcoma of the endometrium and metastatic lobular carcinoma of the breast, the lymphatic vessel density within the tumor was high (MVD=0.419).

Conclusions: The majority of high grade spindle cell sarcomas in this study have a high number of intratumor microvessels, but no or minimal lymphatics. The results suggest that high blood vessel density and lack of lymphatics in sarcoma may account for the fact that they metastasize hematogeneously early to distant sites, instead of through lymphatics to the local lymph nodes.

\section{Spectrum of KOC (K Homology Domain Containing Protein Over-} Expressed in Cancer) Immunostaining among Carcinomas of Different Sites

$S$ Istvanic, GR Fanger, AE Fraire, A Khan, C Li, RK Yantiss. UMass Memorial Health Care, Worcester, MA; Corixa Corporation, Seattle, WA.

Background: We have shown that KOC ( $\underline{K}$ homology domain containing protein over-expressed in cancer), an oncofetal RNA-binding protein, is expressed in pancreatic adenocarcinoma using a novel monoclonal antibody. KOC immunostaining has not been systematically evaluated in other carcinomas and, thus, its specificity for pancreatic cancer is unclear. The aim of this study was to assess KOC expression among carcinomas from different organs in order to establish the spectrum of staining for this marker.

Design: $5 \mu \mathrm{m}$ tissue sections from paraffin-embedded blocks containing lesional and non-neoplastic tissue from 306 carcinomas [breast (26), lung (20), esophagus (14), stomach (25), colorectum (37), anus (10), pancreas (38), liver (18), salivary gland (10), cervix (9), skin (22), bladder (25), kidney (24), thyroid (24), parathyroid gland (3)] were immunostained for KOC using the standard ABC technique. Staining of $>10 \%$ of the tumor was considered a positive result. Any staining of non-neoplastic tissue was also noted.

Results: 127/306 (41.5\%) tumors were positive for KOC (Table 1). 37/38 (97\%) pancreatic carcinomas were positive for this marker, followed in frequency by carcinomas of lung and remaining gastrointestinal tract. Staining was most common in squamous cell carcinoma and adenocarcinomas, whereas other types of carcinoma (e.g. parathyroid gland) were infrequently positive for KOC. All non-neoplastic tissues were negative for KOC in all cases. 
Conclusions: KOC staining is present in many carcinomas and is not a useful discriminator between carcinomas arising in different organs. However, KOC is a highly specific marker for malignancy and thus, may be useful in distinguishing between invasive carcinomas, dysplastic lesions and non-neoplastic tissues in specific situations.

$\begin{array}{lll}\begin{array}{l}\text { Spectrum of KOC Staining Among Carcinomas } \\ \text { Site }\end{array} & \text { Prom Different Organs } \\ \text { Breast } & 2 / 26(8 \%) & \text { Negative cases } \\ \text { Lung } & 12 / 20(60 \%) & 24 / 26(92 \%) \\ \text { Esophagus } & 10 / 14(71 \%) & 8 / 20(40 \%) \\ \text { Stomach } & 15 / 25(60 \%) & 4 / 14(29 \%) \\ \text { Colorectum } & 18 / 37(49 \%) & 10 / 25(40 \%) \\ \text { Pancreas } & 37 / 38(97 \%) & 19 / 37(51 \%) \\ \text { Anus } & 2 / 10(20 \%) & 1 / 38(3 \%) \\ \text { Liver } & 4 / 18(22 \%) & 8 / 10(80 \%) \\ \text { Cervix } & 5 / 9(56 \%) & 14 / 18(78 \%) \\ \text { Bladder } & 9 / 25(36 \%) & 4 / 9(44 \%) \\ \text { Kidney } & 3 / 24(13 \%) & 16 / 25(64 \%) \\ \text { Parathyroid gland } & 0 / 3(0 \%) & 21 / 24(88 \%) \\ \text { Salivary gland } & 0 / 10(0 \%) & 3 / 3(100 \%) \\ \text { Thyroid gland } & 4 / 24(17 \%) & 10 / 10(100 \%) \\ \text { Skin } & 6 / 22(27 \%) & 20 / 24(83 \%) \\ & & 16 / 22(73 \%)\end{array}$

1382 Determination of Evolutionary Mutation Accumulation in Neoplastic Diseases in Pancreas and Bile Duct Brushing Cytology

O Lapkus, O Gologan, YL Liu, PA Swalsky, M Wilson, SD Finkelstein, JF Silverman. Allegheny General Hospital; RedPath Integrated Pathology, Pittsburgh, PA.

Background: Progression of neoplasia characterized by clonal expansion of tumor cells is associated with accumulation of mutational damage. The timing of mutation acquisition could be of value for distinguishing preneoplastic from early and advanced cancer as well as characterize tumor aggressiveness and treatment response. Using quantitative methods applied to microdissected cell clusters selected according to morphologic features, we sought to demonstrate the feasibility and efficacy for determining the time course of mutation accumulation in pancreaticobiliary cytology specimens.

Design: 40 pancreatic and 21 biliary duct brushing cytology specimens were retrieved from the cytology database. Xylene resistant markings were placed on the slide underslide and coverslips removed. Clusters of normal, atypical and malignant cells were manually microdissected and DNA extracted. Mutations (allelic imbalance $[\mathrm{LOH}])$ were quantitatively determined for a broad panel of 15 markers $(1 \mathrm{p}, 3 \mathrm{p}, 5 \mathrm{q}, 9 \mathrm{p}, 10 \mathrm{q}, 17 \mathrm{p}, 17 \mathrm{q}, 21 \mathrm{q}, 22 \mathrm{q})$ and point mutation in $\mathrm{K}$-ras-2 using PCR/ capillary electrophoresis. Time course determination was based on a clonal expansion model with earlier mutation acquisition indicated quantitatively by higher proportion of mutant DNA for a particular marker. The mutational timeline was correlated with cytomorphologic findings (Chi square)

Results: The descending frequency of mutational content in microdissected samples of pancreatic neoplastic lesions was K-ras-2 point mutation (58\%), 3p \& 17q23 (35\%), $5 \mathrm{q}(33 \%), 1 \mathrm{p}(28 \%)$, followed by the remaining molecular markers. The descending percentage of mutated cells followed essentially the same order. K-ras-2 point mutation occured in all but 1 malignant case and contained at least two mutations of the remaining four markers. In the case of biliary cytology specimens, the mutation order was distributed with $17 \mathrm{p} 13,1 \mathrm{p}, 3 \mathrm{p}$ and $5 \mathrm{p}$ representing early mutations.

Conclusions: The molecular pathogenesis of pancreatic and biliary neoplasia involves a narrow and distinct subset of defined mutations that includes the studied markers occurring early in tumorigenesis. K-ras- 2 point mutation is most frequently acquired early and is associated with a more rapid mutation acquisition in pancreatic brushing specimens. The time course of mutation accumulation can be determined in cytology specimens and can be incorporated into the diagnostic characterization of the neoplasm.

1383 Distinguishing Fibrovascular Septa from the Extravascular Patterned Matrix of Vasculogenic Mimicry

AY Lin, S Kadkol, J Pe'er, S Setty, D Majumdar, L Leach, AJ Maniotis, R Folberg. University of Illinois at Chicago, Chicago, IL; Hadassah University Hospital, Jerusalem, Israel.

Background: The histogenesis of $\mathrm{PAS}^{+}$(laminin-rich) extravascular patterned matrix in highly invasive melanomas is controversial. Molecular analyses of matrix components suggests these patterns are generated by invasive tumor cells (vasculogenic mimicry). Some observers, however, consider these patterns to represent fibrovascular septa that originate predominantly from a stromal host response.

Design: Histologic sections of 234 primary uveal melanomas were stained by PAS and trichrome to identify the frequency distribution of looping patterns and any association with outcome. The circumference thickness of trichrome ${ }^{+}$and $\mathrm{PAS}^{+}$loops was compared by quantitative morphometry. Adjacent sections from 13 additional primary uveal melanomas that contained $\mathrm{PAS}^{+}$loops but not thick trichrome ${ }^{+}$loops were stained for collagen I. RQ-PCR was performed on RNA extracted from lowinvasive and high invasive primary uveal melanoma cells for the expression of collagen I (COLIA2). 3D rafts composed of pure type I collagen were seeded with highly invasive or poorly invasive tumor cells and the rafts were photographed and weighed over 7 days.

Results: Trichrome ${ }^{+}$loops were identified in 24 of 234 cases (10\%) compared with $\mathrm{PAS}^{+}$loops in 131 cases $(56 \%)$. Although the detection of $\mathrm{PAS}^{+}$loops was associated with death from metastatic melanoma $(\mathrm{p}<0.0001)$, there was no association between trichrome ${ }^{+}$loops and tumor-associated mortality $(\mathrm{P}=0.5702)$. Trichrome ${ }^{+}$loops were significantly thicker than PAS-positive loops $(\mathrm{P}=0.0001)$. By immunohistochemistry, collagen I was detected in thin $\mathrm{PAS}^{+}$patterns in $8 / 13$ cases and within tumor cell cytoplasm. RQ-PCR revealed a 97x increase in expression of collagen I by highly invasive primary melanoma cells compared with poorly invasive cells. Floating rafts composed of type I collagen were degraded by highly invasive melanoma cells but not by poorly invasive tumor cells.

Conclusions: Fibrovascular septa are rare and prognostically insignificant in uveal melanomas because highly invasive tumor cells degrade collagen I. By contrast, the extravascular patterned matrix is associated with increased mortality. Limited distribution of collagen $I$ in the extravascular patterned matrix may result from its synthesis and degredation by tumor cells, independent of a host stromal response. Supported by NIH grant EY10457

1384 Intra-Tumor Depletion of CD4+ Cells Unmasks Tumor Immunogenicity Leading to Rejection of Established Tumor

W Liu, P Yu, Y Lee, T Krausz, W Recant, Y Fu. The University of Chicago Hospitals, Chicago, IL.

Background: The frequent observation of $\mathrm{T}$ cell infiltration into cancer tissues indicates that immune recognition of cancer occurs. However, it is exceptional for such tumor infiltrating $\mathrm{T}$ cells to induce the spontaneous rejection of established tumors. Many studies present the possibility of a local environment at the tumor site may play critical roles for preventing the immunological destruction of antigenic tumors. The Foxp3 expressiong CD4+CD25+ T cell subset could severely limit the efficacy of vaccine-induced antitumor immune responses through inhibiting the CD8+ T cells. We sought to determine whether abrogating the effects of CD4+CD25+ T cells at the tumor site could be an effective approach to enhance anti-tumor immunity, leading to rapid rejection of well-established tumors.

Design: Human breast cancer samples (20 DCIS and 20 infiltrating ductal carcinomas) were stained with anti-CD4, CD8, CD25 and FOXP3 antibodies. The Ag104 fibrosarcoma and Ag104 cell line expressing murine H-2Ld were injected to mice. $\mathrm{CD} 4+\mathrm{CD} 25+$ cells were isolated from the murine tumor and the regulation of CD8+ cells by CD4+CD25+ cells was studied. Intra-tumoral injection of anti-CD4 and CD25 antibodies was carried out. We analyzed the changes of tumor sizes, intratumoral CD8+ number, inflammatory and anti-inflammatory cytokine expression levels and foxp3 gene expression.

Results: The vast majority of lymphocytes are CD8+ T cells in breast DCIS, however, the CD4+ T cell population increased dramatically in infiltrating ducal carcinomas Similar phenomena were demonstrated in murine model. CD4+CD25+T cells suppressed the proliferation and interferon- $\gamma$ production of CD8+ T cells at the local tumor site. Blockade of the effects of IL-10 and TGF- $\beta$ could partially reverse the suppression imposed by the CD4+ cells. Local depletion of CD4+ cells leads to the eradication of well-established tumors and to the development of long-term antitumor memory.

Conclusions: There was a selective accumulation of $\mathrm{CD} 4+\mathrm{CD} 25+\mathrm{T}$ cells inside malignant tumors. CD4+CD25+ T cells primarily mediated a suppressive environmen inside the tumor and abrogated the effector function of CD8+ T cells. Local intra-tumoral depletion of these regulatory $\mathrm{T}$ cells unmasks the immunogenicity of tumor and reverses CTL tolerization leading to the rapid rejection of well-established tumors. T regulatory cells could be one important local factor to suppress immune responses against a strong tumor antigen leading to progressive growth of cancer in the immune competent hosts.

\section{Overexpression of RNase L Inhibits Tumor Growth in Fibrosarcoma} in Nude Mice

WW Liu, R Gollapalli, A Zhou. Case Western Reserve University, Cleveland, OH; Cleveland State University, Cleveland, $\mathrm{OH}$.

Background: RNase L is one of the key enzymes involved in interferon signal transduction pathways. It mediates the antiviral and anti-proliferative functions of interferon. RNase L has also been found to exert proapoptotic activity independent of interferon. However, the exact function of RNase $\mathrm{L}$ in tumorigenesis is unknown. This study was designed to study the role of RNase $\mathrm{L}$ in tumor growth using a nude mice model.

Design: To directly measure the effect of RNase L on tumor growth in the absence of other IFN-induced proteins, human RNase L cDNA was stably expressed in P-57 cells, an aggressive mouse fibrosarcoma cell line. Several clonal cell lines were isolated in which overexpression of RNase L was 20-30 fold of the endogenous level. Groups of three nude mice were injected subcutaneously with either the human RNase L overexpressing clones (PL-RNase L) or control cells transfected with an empty vector (PL-vector). Tumor formation by these two cell lines was monitored by measuring tumor volume.

Results: In the RNase L+ group, tumor formation was significantly delayed and the tumors grew much slower compared to the control group. Morphologically, the RNase L+ tumor cells changed from spindle cells with a herringbone pattern to more polygonal cells, demonstrated more discohesion, and showed increased areas of tumor necrosis. Interestingly, after 5 weeks, the growth of RNase L+ tumors started to accelerate. Eventually tumors in both groups reached the same size, corresponding to the fact that the expression of RNase L was completely shut down in RNase L+ group. 


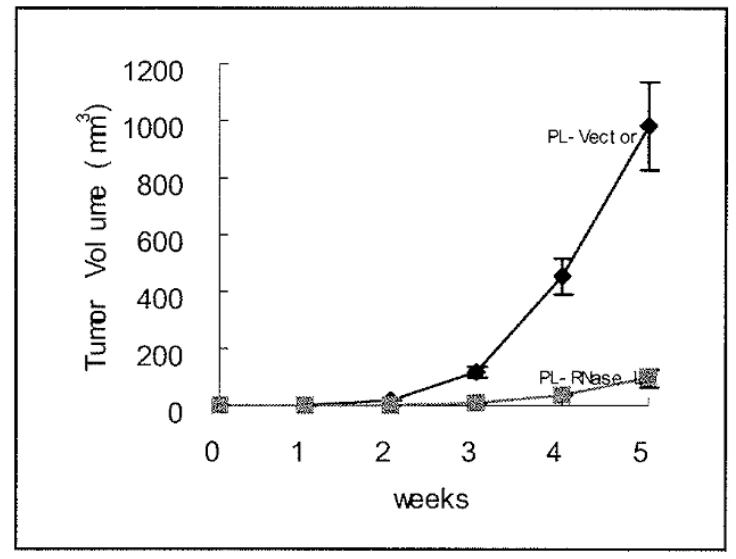

Conclusions: Overexpression of RNase L significantly inhibits the tumor growth, suggesting that RNase L plays a critical role in growth regulation of fibrosarcoma in nude mice.

\section{Elevated Expression of BP1 in Both Invasive and Metastatic} Inflammatory Breast Cancer

YG Man, AM Schwartz, P Levine, PE Berg. AFIP, Washington, DC; George Washington University, Washington, DC.

Background: BP1, a homeobox gene, is expressed in an increasing percentage of hyperplastic, in situ, and invasive breast lesions, in contrast to its sparse detectability in normal human breast tissues. This study investigates the immuno-expression of BP1 in inflammatory breast cancer (IBC), a relatively rare but very aggressive form of breast cancer characterized by extensive lympho-vascular invasion.

Design: Paraffin-embedded tissue sections from 40 cases of IBC, seven with paired metastatic lymph nodes, were assayed immunohistochemically for BP1 and for a panel of markers specific to epithelial cells, blood vessels, and lymphatic channels. Results: BP1 immunoreactivity was identified in all cases of IBC with intensitie ranging from focal to diffuse and strong. Adjacent benign ducts were immunoreactive in a minority of cases, similar to our previous results. Immunoreactivity of metastatic tumors was equal to or greater than the staining reactivity present in the primary breast carcinoma. Carcinoma within lymphatic channels was uniformly positive for BP1 immunoreactivity. The percentage and distribution of immunoreactivity in the cohort of IBC cases were greater than a comparison cohort of non-IBC ductal carcinomas.

Conclusions: BP1 expression was identified uniformly in IBC and its metastases. Current and previous studies suggest that BP1 may act as an oncogene promoting tumor progression and metastasis.

1387 EBV Infection of NOD/SCID Mice Reconstituted with Human Hematopoitic CD34+ Cells: A Potential In Vivo Model for EBV Induced Lymphoproliferative Disorder (LPD) in Immunodeficient Patients

GJ Netto, M Islas-Ohlmayer, R Domiati-Saad, A Padgett-Thomas, MW Melkus, PD Cravens, MP Martin, RS Scott, JW Sixbey, JV Garcia. Baylor University Medical Centre, dallas, TX; University of Texas Southwestern Medical Centre, Dallas, TX Louisiana State University Health Sciences Center, Shreveport, LA

Background: EBV-induced LPD is a serious complication of immune deficiency Inadequate T-cell suppression allows the outgrowth of transformed cells resulting in B-cell lymphomas. The mechanism by which EBV transforms B-cells is not well understood. An in vivo model that recapitulates EBV infection and its association with B-cell lymphomas is currently lacking. Experimental EBV infection of NOD/ SCID mice reconstituted with human CD34+ stem cells could serve as such model. Design: NOD/SCID mice engrafted with human CD34+ cells and reconstituted mainly with human B-lymphocytes were used. Mice were infected with EBV tagged with the enhanced green fluorescence protein (EGFP). The latter, could be traced in developing tumors using UV light and detected in peripheral blood (PB) and bone marrow (BM) by flow cytometry (FCM). Paraffin embedded tissue samples were evaluated histologically (H\&E); immunohistochemically (CD20,CD79a,CD30,CD5 and Kappa/ Lambda light chains) and by In situ hybridization (EBER). PB EBV viral loads were evaluated at weekly intervals by Real Time PCR.

Results: All Mice PB samples demonstrated high EBV DNA viral load by week four. All infected mice developed visible tumors in multiple organs most prominently in the spleen. All tumors had morphologic and immunophenotypic features consisten with diffuse large B cell lymphoma, immunoblastic type, WHO classification (CD79a+, CD20+, CD30+, EBER+ and 30\% CD5+). all tumors were light chain restricted by immuhistochemistry. Tumor cells expressed EBNA1, LMP1 and LMP2a by RT-PCR (type II pattern of latent EBV gene expression). Culture of cells from bone marrow and spleen of infected mice yielded EBV+ lymphoblastoid cell lines (LCLs) that were positive for CD79a , CD19, CD5, and CD30 by FCM. Finally, EGFP-tagged EBV enabled us to detect infected EGFP+ cells and of EGFP+ tumors All developing tumors demonstrated strong florescence that could be visualized under UV light. PB and BM samples also revealed EGFP expression by FCM.
Conclusions: Our data demonstrate that NOD/SCID mice transplanted with human CD34+ cells are susceptible to infection by EBV and accurately recapitulates important aspects of EBV pathogenesis.

1388 Colonic Adenocarcinomas Show Increased Ratio of Saturated to Unsaturated C18 Fatty Acids When Compared to Normal Colonic Mucosa

D Rakheja, P Kapur, MP Hoang, MJ Bennett. University of Texas Southwestern Medical Center, Dallas, TX.

Background: Mammalian fatty acid synthase (FASE) overexpression has been shown in a number of human malignancies, including colonic adenocarcinoma. Since FASE synthesizes only saturated fatty acids, and since humans do not have the enzymatic machinery to convert these saturated fatty acids to polyunsaturated fatty acids, we hypothesize that cancer cells have a greater proportion of long-chain saturated fatty acids.

Design: Fresh tissue samples from 13 colonic adenocarcinomas and adjacent normal colonic mucosa were obtained from surgical specimens. The samples were homogenized followed by one-step lipid extraction and methyl esterification. Palmitic, palmitoleic, stearic, oleic, and linoleic acids were then measured by gas chromatography-mass spectrometry in selective ion mode. Statistical analyses were performed on Microsoft Excel spreadsheet using 1-tailed Student's t-test.

Results: There was a relative abundance of stearic acid in colon carcinoma as compared to normal colonic mucosa, as shown in the Table.

Conclusions: Our study shows unequivocal increase in saturated $\mathrm{C} 18$ fatty acid (stearic acid) in colonic adenocarcinoma compared to adjacent normal colonic mucosa. This may create significant alterations in physical and biological properties of cancer cells, providing avenues for therapeutic interventions.

$\begin{array}{llll}\text { Fatty acid ratio } & \begin{array}{l}\text { Normal tissue } \\ (\mathbf{m e a n} \pm \mathbf{s d})\end{array} & \begin{array}{l}\text { Tumor tissue } \\ (\mathbf{m e a n} \pm \mathbf{s d})\end{array} & \text { p-value } \\ \text { Palmitic:Palmitoleic } & 7.25 \pm 2.70 & 7.52 \pm 2.71 & 0.406 \\ \text { Stearic:Oleic } & 3.92 \pm 1.50 & 6.44 \pm 1.95 & 0.001 \\ \text { Stearic:Linoleic } & 1.13 \pm 0.19 & 1.81 \pm 0.30 & <0.001 \\ \text { Stearic:Oleic+Linoloeic } & 0.85 \pm 0.16 & 1.39 \pm 0.23 & <0.001 \\ \text { Palmitic+Stearic: } & 1.34 \pm 0.19 & 1.90 \pm 0.24 & <0.001\end{array}$

Palmitoleic+Oleic+Linoloeic

1389 A Distinctive Association of Activated mTOR Pathway in Human Epithelial Tumors: Akt and 4EBP1 as Targets of Cell Signalling

$S$ Ramon y Cajal, C Iglesias, J Jimenez, S Rodriguez, J Castellvi, J Baselga, F Rojo. Vall d'Hebron University Hospital, Barcelona, Spain.

Background: Activation of the PI3-K/Akt/mTOR signal transduction pathway contributes to the development and progression of tumors by prevention of apoptosis and deregulation of the cell cycle. Dissecting the molecular events of this pathway may provide instrumental knowledge to understand a different potential behaviour in tumors, mechanisms of activation of this signalling cascade and implications for the development of therapeutic PI3K/Akt/mTOR inhibitors.

Design: We have analysed 247 paraffin-embedded human malignant epithelial tumors: 112 ovary, 56 breast, 25 gastric, 25 colon, 19 prostate and 10 pancreas, with a complete immunohistochemistry profile including multiple phosphorylated (p) downstream proteins: pAkt, p4EBP1, pp70S6K and pS6. The levels of expression were evaluated as percentage and intensity of stained tumor cells (Hscore).

Results: Globally, there was a significant correlation between the levels of pAkt, p4EBP1, pp70S6K and pS6 (Spearman's test, p<0.001). Interestingly, high pAkt expression was consistently observed in all tumor types whereas the downstream effectors presented higher activation in pancreatic, breast and prostate than in gastric and colon carcinomas (table, Pearson Chi square, $\mathrm{p}=0.005$ ). Activated mTOR downstream effectors were mainly detected in poor differentiated tumors.

Conclusions: Detection of p proteins of the Akt/mTOR pathway is feasible in a variety of tumor types. Our findings suggest that level of in vivo activation of Akt is significant in a variety tumor types but activation of mTOR downstream proteins could be higher in different tumor types by alternative mechanisms: both Akt and 4EBP1 can be expressed concomitantly or independently in tumors and be associated with different signalling pathways that result in proliferation, apoptosis and cell growth. The oncogenic alterations that mediate and drive these labyrinthine cellular pathways have to be determined. Type of tumor

$\begin{array}{lllllll} & \text { Ovary } & \text { Breast } & \text { Gastric } & \text { Colon } & \text { Prostate } & \text { Pancreas } \\ & \mathrm{N}(\%) & \mathrm{N}(\%) & \mathrm{N}(\%) & \mathrm{N}(\%) & \mathrm{N}(\%) & \mathrm{N}(\%) \\ \mathrm{N} & 112 & 56 & 25 & 25 & 19 & 10 \\ \text { pAkt } & 28(25) & 22(39.5) & 8(32) & 8(32) & 11(57.9) & 5(50) \\ \text { p4EBP1 } & 82(73.2) & 31(55.3) & 0(0) & 1(4) & 6(31.6) & 4(40) \\ \text { pS6 } & 22(19.6) & 15(26.8) & 2(8) & 0(0) & 3(15.8) & 5(50) \\ \text { pp70S6K } & 48(42.9) & 14(25) & 0(0) & 0(0) & 7(36.8) & 3(30)\end{array}$

1390 A Mouse Model of Gastroinstestinal Stromal Tumor

BP Rubin, ML Comstock, JP Scott-Browne, MR Tanas. University of Washington Medical Center, Seattle, WA.

Background: Gastrointestinal stromal tumors (GISTs) are the most common mesenchymal tumors of the gastrointestinal tract. Most GISTs harbor activating mutations in Kit, a receptor tyrosine kinase (RTK). Rare families have germline activating Kit mutations and family members with these mutations develop interstitial cell of Cajal (ICC) proliferations and GISTs. We have constructed a mouse model harboring a germline activating Kit mutation, which mimics the human familial GIST syndrome.

Design: We have used a genetic knock-in strategy to replace one normal Kit allele with a mutant Kit allele that harbors a K641E mutation. This mutant allele is analogous to a mutation seen in a single human familial GIST syndrome family. Mice harboring 
this allele were interbred and monitored for the development of abdominal masses or distended abdomens. Mice developing masses or distended abdomens were sacrificed and necropsied per protocol. Tissues were examined by routine histology and by immunohistochemistry with antibodies to Kit, smooth muscle actin, desmin, and CD34. Tumor lysates were immunoprecipitated with an antibody to Kit and the immunoprecipitate was fractionated on a polyacrylamide gel and western blotted with an anti-phosphotyrosine antibody. Flow cytometry of bone marrow was performed using an anti-Kit antibody.

Results: Mice harboring these mutations are viable and generated at the expected Mendelian frequencies. Heterozygous mice are viable and phenotypically normal. Homozygous mutant mice (Kit ${ }^{\mathrm{K} 641 \mathrm{E} / \mathrm{K} 641 \mathrm{E}}$ ) express Kit at greatly reduced levels as evidenced by loss of function phenotypes, including lack of skin pigmentation and sterility. Low Kit expression was confirmed by flow cytometry of whole bone marrow with an anti-Kit antibody. However, $100 \%$ of the homozygous mice develop gastrointestinal pathology beginning at 4 weeks of age in the form of ICC proliferations of the distal esophagus, stomach, and rectum and GISTs of the cecum. Kit immunoprecipitation reveals the GISTs to have very high levels of phosphorylated/ activated Kit

Conclusions: We have developed a mouse GIST model in which $100 \%$ of the mice develop ICC proliferations and GISTs. This model will be useful in understanding the pathogenesis of GISTs as well as serving as an excellent pre-clinical model to test the efficacy of therapies which target Kit.

1391 DNA Methylation Regulates Galectin-3 Expression in Various Cell Lines and in Pituitary Tumors

KH Ruebel, L Jin, BW Scheithauer, K Kovacs, RV Lloyd. Mayo Clinic, Rochester, MN; St. Michaels Hospital, Toronto, ON, Canada.

Background: The LGALS3 gene is expressed in many tissues and increased expression has been associated with some malignant tumors. Galectin-3 (Gal-3) protein was recently observed in subsets of pituitary tumors, and there was increased expression in pituitary carcinomas. The mechanisms regulating Gal-3 expression are unknown. Design: We analyzed the promoter region of the LGALS3 gene by methylation specific PCR (MS-PCR) and DNA sequencing in various tumor cell lines including breast carcinomas $(n=3)$, thyroid carcinomas $(n=3)$, a pituitary adenoma and HeLa cell lines. Twenty-three pituitary tumors were also examined. Gal-3 protein expression was also analyzed by Western blotting.

Results: In most cell lines, the LGALS3 gene was unmethylated and there was expression of Gal-3 protein. The SKBR3 breast carcinoma cell line was methylated and did not express Gal-3. Treatment of cells with 5'-azacytidine resulted in expression of Gal-3 protein and mRNA in SKBR3 cells. Among pituitary tumors, $30.4 \%$ were methylated. The pituitary tumors frequently associated with development of pituitary carcinomas (prolactin and ACTH) were all unmethylated.

Conclusions: Epigenetic inactivation of LGALS3 is an important mechanism regulating Gal-3 expression. Analysis of Gal-3 methylation may be a useful diagnostic marker.

1392 The Ataxia-telangiectasia Gene Product Is Required for Genomic Stability Following Labile Ferric Iron Exposure

RE Shackelford, RP Manuszak, $S$ Wang, $M$ Lowery-Norberg, A Chen. Louisiana State University, Shreveport, LA; Iowa Cancer Research Foundation, Urbandale, IA. Background: Ataxia-telangiectasia (A-T) is a rare autosomal recessive disorder characterized by immune dysfunction, progressive cerebellar ataxia, and an 1,000X elevated cancer incidence. The gene mutated in A-T, ATM, has been cloned and exhibits oxidant and ionizing radiation-induced kinase activity. In culture, cells from individuals with A-T exhibit increased genomic instability and oxidant sensitivity, compared to normal cells. Since labile iron plays a major role in toxic oxygen free radical production via Fenton catalysis, we hypothesized that ATM is necessary in the maintenance of genomic stability and cellular viability following labile iron exposure.

Design: The effect of labile ferric iron on normal, A-T, and A-T cells which express wild type ATM-protein was examined by colony-efficiency forming assay and analysis of double-stranded DNA breaks in metaphase cell preparations. The effect of the iron chelators desferal, apoferriton, quercetin, and epigallocathechin-3 gallate were also examined on A-T and normal cell viability. Additionally Atm-deficient mice were treated with interperitoneal desferal injection and the effect on their body weight was recorded.

Results: We show that modest increases in labile ferric iron (a 100nM increase) significantly lowered A-T, but not normal cell viability. Labile iron also increased A$\mathrm{T}$, but not normal cell dsDNA breaks at low concentrations. These effects were absent in A-T cells expressing functional ATM protein. Iron chelators also increased A-T, but not normal cell genomic stability and viability, with and without exogenous oxidative stress. Last, interperitoneal injection of Atm-deficient mice with the iron chelator desferal increased Atm-deficient mouse weight.

Conclusions: ATM is required for the maintenance of cell viability and genomic stability in the face of low labile ferric iron levels. Additionally iron chelators increase A-T cell genomic stability and viability, and Atm-deficient mouse weights. Iron chelators may therefore prove useful in the treatment of A-T. Also, since iron plays a central role in several diseases, such as hemochromotosis and Parkinson's disease, our data indicates that ATM function may prove important in these disease processes.
1393 Lens-Like Expression of Crystallins in the Corneas of Cats with Mucopolysaccharidoses I and VI

183 Villa, J Alroy, M Haskins. New England Medical Center - Tufts University School of Medicine and School of Veterinary Medicine, Boston, MA; University of Pennsylvania, School of Veterinary Medicine, Philadelphia, PA.

Background: Lysosomal storage disorders represent a group of over 45 distinct genetic illnesses, with an estimated incidence of 1/7,000 live human births. Lysosome catabolize cellular and extracellular macromolecules and provide substrate for resynthesis of cellular and extracellular molecules. A common biochemical feature of lysosomal storage is the accumulation of undegraded or partially degraded substrates within the lysosomes leading to cell dysfunction. A phenotypic characteristic of humans and animals affected with mucopolysaccharidosis (MPS) I (Hurler disease), MPS IV-A (Morquio A disease), MPS VI (Maroteaux-Lamy disease), and MPS VII (Sly disease) is cloudy cornea. Current morphologic explanations for this phenomenon include the presence of enlarged secondary lysosomes packed with storage material, and the disorganization of collagen in the corneal matrix; however, the molecular basis remains unknown.

Design: In this study we utilized differential display proteomics with "fused and super-fused" images using the software Delta 2D (Decodon.com) to compare corneas of cats affected with MPS I and VI or normal age/matched littermates.

Results: A total of 2729 spots were detected and 53 spots were found specifically upregulated (over three-fold). The top twelve upregulated spots were punched out of the gels, digested with trypsin and fed into a micro HPLC, tandem mass spectrometer (uLC-MS-MS; LCQ-DECA Thermo Finnegan). Sequence analysis and database search was performed with the software Sequest and it showed a family of proteins not previously identified in the cornea of humans or animals; the lens proteins crystallin alpha, beta and gamma.

Conclusions: We hypothesize that cell trafficking dysregulation caused by the truncated gene product of the enzymes alpha-L-iduronidase and $N$-acetylgalactosamine 4-sulfatase per-se or the accumulation of dermatan sulfate and heparan sulfate in MPS-I, and dermatan sulfate and chondroitin 4-sulfate in MPS VI may have altered the gene regulation of the crystalline family leading to its production and deposit in the cornea of the affected animals.

1394 Dynamic Duo Orchestrates the Double-Edged Sword

EX Wei, M Sasaki, VL Dawson, TM Dawson. Louisiana State University, Shreveport, LA; Johns Hopkins University, Baltimore, MD.

Background: Nitric oxide (NO) plays a critical role in neuronal signaling, cerebral ischemia, neuronal injury and neurodegenerative disorders. Neuronal nitric oxide synthase (nNOS) accounts for the majority of actions of NO in the central nervous system (CNS). nNOS mRNA and protein is significantly upregulated by calcium influx under ischemic or injurious conditions. Multiple promoters control nNOS transcription. The major promoter resides at the proximal promoter and confer calcium responsiveness. Promoter analysis identified several key regulatory sequences of the main promoter, including a monomeric nuclear receptor response element (NRE) and two cAMP/ $\mathrm{Ca}^{2+}$ response elements (CREs). CRE binding protein (CREB) interacts with CREs in neurons and steroidogenic factor 1 (SF-1) binds to NRE in pituitary gonadotropes. In the CNS, the transcription factors that bind to the NRE needs to be clarified.

Design: Rat embryonic cerebral cortical neurons were collected and cultured. Various nNOS reporter constructs and overexpression plasmids were transfected. The reporter activities were analyzed. Gel shift assays (GSA) were performed using neuronal nuclear extracts.

Results: Deletion and mutation of the NRE dramatically diminished nNOS calcium inducibility. Although only exogenous SF-1 significantly increased nNOS promoter activity, the nature of the protein was not definitively determined, as antibodies against known monomeric nuclear receptors (NRs), including SF-1, ERR $\alpha, \beta, \gamma$, Nurr1 and Nur77, could not disrupt the DNA/protein complexes in GSA. It is likely to be a SF-1-like NR family member. It cooperatively interacts with CREB and the NR coactivators SRC-1 and CBP, and regulates nNOS transcription by calcium influx. COUP-TFI, a transrepressor, is a dimeric NR essential for neuron development and capable of binding to monomeric and dimeric NREs. COUP-TFI binds to the same nNOS NRE in GSA, but with less affinity and forms its own distinct band. It recruits corepressor SMRT and suppresses nNOS transcription. It dose-dependently competes with SF-1-like protein and vice versa.

Conclusions: NRE is the fourth calcium response element found so far. Identification of the SF-1-like protein is still in progress. This potential novel nuclear receptor and COUP-TFI form a dynamic duo, in conjunction with CREB, orchestrating the fine transcriptional regulation of nNOS, a double-edged sword, in CNS under normal and stimulated conditions. They may contribute to NO actions in stroke, neuronal injury and other CNS diseases.

1395 Role of p53, IGFR and EGFR in Clusterin Expression in Rectal Cancers $J$ Willis, A Das, A Chak, K Ferguson, L Mayo, D Dawson, D Boothman. University Hospitals of Cleveland; Case Western Reserve University, Cleveland, $\mathrm{OH}$.

Background: Clusterin is a ubiquitous cellular glycoprotein that has been implicated in a number of pathways of carcinogenesis, as well as in cellular systems associated with cell injury leading ultimately to cell death. Studies in different models and organ systems have recently recognized two differing isoforms with dramatically different functions. A cytoplasmic/secretory form of the clusterin protein that has cytoprotective effects and is associated with tumor progression. Additionally, a nuclear form has recently been identified whose activation is related to cell death processes and turnover of dying cells. Studies performed on cell culture models in our laboratory have implicated p53 and IGFR pathways in regulating clusterin formation and function and excluded EGFR pathway as an important modulator of clusterin 
expression. To further explore this hypothesis we investigated immunohistochemical staining for nuclear and cytoplasmic clusterin expression in a cohort of rectal cancers and investigated its co-expression with p53, EGFR and IGFR.

Design: Representative tissue blocks from 37 rectal cancer resections retrieved from our surgical pathology department archive were used. These neoplasms were immunostained using commercially available antibodies for nuclear and secreted forms of clusterin, p53, phosphorylated EGFR and non-phosphorylated IGFR. Each slide was scored independently and results were expressed in terms of percentage of tumor cells staining $\mathrm{x}$ intensity.

Results: Median score and range for each stain was as follows: nuclear clusterin 10 [0-270]; cytoplasmic clusterin 30 [0-240]; p53 210 [10-270]; IGFR 180 [0-270]; EGFR-Phos 120 [0-270]. Using a Spearman R test a significant negative correlation was identified between expression of nuclear clusterin and IGFR as well as nuclear clusterin and p53 staining. A positive correlation was identified between cytoplasmic clusterin and cytoplasmic EGFR-Phos. No correlation was found between nuclear and cytoplasmic clusterin.

Conclusions: These findings corroborate our previously reported cell culture experiments of an association between nuclear clusterin and p53 status in tumor cells. These results support the concept of a pivotal role of IGFR in the modulation of nuclear clusterin expression. The data also suggest that in rectal cancer EGFR activation may signal secretory clusterin expression.

\section{Involvement of CD5 and CD10 in the Development of Choroid Plexus Papillary Neoplasm}

SX Yan, YL Liu, M Tung, JF Silverman. Allegheny General hospital, Pittsburgh, PA Background: Choroid plexus papillomas (CPPs) are benign, relatively uncommon neoplasms that occur especially in children. The epithelial nature of the normal choroid plexus and choroid plexus neoplasm has been confirmed by previous studies demonstrating positive expression of epithelial markers such as cytokeratins and EMA. CD5 and CD10 are membrane glycoproteins used extensively in working up lymphoproliferative disorders, but have also been shown to be expressed in a variety of normal epithelium and carcinomas. However, the expression of CD5 and CD10 in the choroid plexus papillary neoplasms have not been studied.

Design: 19 cases including 8 CPPs and 11 normal choroid plexus were retrieved from the laboratory database. Immunostaining with antibodies to CD5 and CD10 was performed on paraffin-embedded tissue. Immunostains were performed on an automated immunostainer with appropriate positive and negative controls.

Results: CD5 immunoreactivity was demonstrated in 7/8 (88\%) of choroid plexus papillomas and none of the eleven normal choroid plexus studied. CD10 was positive in $3 / 8(38 \%)$ of choroid plexus papillomas and in only $1 / 11(9 \%)$ of normal choroid plexus.

Conclusions: Our results showed that CD5 and CD10 is expressed in choroid plexus papilloma, but not in normal choroid plexus. The expression of CD5 and CD10 may be involved in the pathogenesis of choroid plexus neoplasm and can also be useful in separating normal choroid plexus from choroid plexus papillomas, especially when very limited material is present in the brain biopsy.

\section{Effets of Retinoic Acid on Promyelocytic Leukemia Protein- Induced Growth Suppression and Cell Death in Solid Tumor Cells}

E Yu, SH Son, EK Choi, H Lee, J Choi. University of Ulsan Collge of Medicine Asan Medical Center, Seoul, Korea.

Background: The promyelocytic leukemia protein (PML), involved in the pathogenesis of acute promyelocytic leukemia (APL), is a co-activator in p53 tumor suppressive functions. The ability of the PML to inhibit growth and to induce cell death of solid tumor cells, however, has not been determined. As a therapeutic agent in APL, retinoic acid (RA) displays antiproliferative effects in solid tumor cells of the breast, the prostate and the lung by promoting apoptosis.

Design: To evaluate the tumor suppressor ability of the PML in solid tumors, we first assayed the antiproliferative activities of the PML by using replication-deficient recombinant PML adenovirus (Ad-PML) and compared them with those of p53 in four liver cancer cell lines. Next, we examined whether RA has synergistic effects on the PML-induced tumor cell suppression or not

Results: Following infection of cells with Ad-PML, in vitro growth curve analysis showed that the overexpressed PML initially induced a substantial G1 cell cycle arrest and triggered massive cell death in all tested cell lines, irrespective of their p53 status. The PML-induced cell death decreased by about $30 \%$ in the presence of a broad caspase inhibitor, zVAD. The cell death effect of the PML was higher than that induced by $\mathrm{p} 53$ over a longer period of time. As with p53, the overexpression of the PML was closely related to up-regulation of p21 and the decrease of cyclin D1 expression. Unexpectedly, RA antagonized rather than enhanced the PML-triggered cell death. RA enhanced the expression of adenovirus-CMV-promoted PML at both transcription and protein levels within $12 \mathrm{~h}$ after the treatment. However, the PML was significantly degraded in the presence of RA at days 3-5 postinfection. The PML degradation was also observed in SK-BR3 breast cancer cells treated with RA. RA treatment reduced retinoic acid receptor (RAR) expression. Addition of LLnL, a proteasome inhibitor, blocked RA-induced decreases in the PML and RAR proteins. Conclusions: Our findings strongly support the hypothesis that the PML acts as a strong independent cell death inducer and that RA conversely abolishes the therapeutic effects of the PML through proteasomal degradation of the protein.
1398 Expression of Transforming Growth Factor b1 and It's Receptor, Smad and JNK in Colorectal Adenocarcinomas

G Yue, M Tung, YL Liu, JF Silverman. Allegheny General Hospital, Pittsburgh, PA. Background: Colorectal adenocarcinoma is one of the most common cancers and leading cause of death in United States. Transforming growth factor b1 (TGFb1), a potent inhibitor of cell growth, activates Smad and JNK proteins by binding it's receptor. Smad and JNK are nuclear transcriptional activators regulating gene transcription. The resulting repression of c-myc and induction of cyclin-dependent kinase inhibitors leads to G1 phase cell cycle arrest. Previous studies indicated that decreased expression of Smad and JNK is associated with poor prognosis in a number of carcinomas. However, the correlation of TGFb1 and it's signal transduction 's expression and prognosis in the colorectal adenocarcinoma has not be investigated. Design: A total of 20 cases of primary and metastatic colorectal adenocarcinomas were retrieved from the hospital data base. In the primary colorectal adenocarcinoma without lymph node and distant metastasis, there are five each of poorly and well differentiated adenocarcinomas. Five cases of colorectal adenocarcinoma with positive lymph nodes and five metastatic colonic adenocarcinoma with liver metastasis were selected. Immunostaining for TGFb1, TGFb1 receptor, Smad2, and JNK were performed on an automated immunostainer with appropriate positive and negative controls. The intensity of immunostain was measured and classified into four scale titer with negative (score:0), weak (score:1), strong (score:3) and between (score:2) by two observers. Statistical analysis was performed with Chi-Square test.

Results: TGFb1, TGFb1 receptor, Smad, and JNK were observed in all adenocarcinomas in this study. The immunostaining patterns are as follow. TGFb1 and TGFb1 receptor show membrane and cytoplasmic pattern. Smad 2 shows nuclear pattern. JNK shows nuclear and cytoplasmic pattern. There is no statistic difference in the expressions of TGFb1, TGFb1 receptor, Smad and JNK in the primary and metastatic colon adenocarcinomas.

Conclusions: Our results indicate that the expression of TGFb1, TGFb1 receptor, Smad, and JNK can not be used as a prognostic factor for colorectal adenocarcinomas and support the hypothesis that the aggressive behaviors of colorectal carcinomas may be associated with the mutations rather than the decreased expression of TGFb1 and it's signal transduction system.

1399 Induction by Selenium of Apoptosis in Endometrial Cancer Cells Is Coupled with an Increase in the Level of Active Caspase-3

$P$ Zhang, C Zhang, CJ Sung, F Liu, WD Lawrence. Women \& Infants Hospital, Brown Medical School, Providence, RI.

Background: Selenium (SE), a constituent of antioxidant enzymes, has been proposed as a chemopreventive and chemotherapeutic agent for prostate and several other cancers; however, no information is available concerning its effect in endometrial carcinoma. Many chemotherapeutic agents inhibit cancer growth by inducing apoptosis. Caspase-3, a cellular protease, plays a major role in apoptosis. It is expressed in cells as an inactive $32 \mathrm{kDa}$ precursor which, during apoptosis, forms an active $11 \mathrm{kDa}$ subunit and an inactive $20 \mathrm{kDa}$ peptide; the latter breaks down into an active $17 \mathrm{kDa}$ subunit. The aim of this study is to investigate the effects of SE on cell growth, apoptosis, and the roles of different forms of caspase- 3 in cultured endometrial cancer cells (EC).

Design: EC (American Type Culture Collection, Manassas, VA) was incubated with different concentrations of sodium selenite $(0 \mu \mathrm{M}, 2 \mu \mathrm{M}, 4 \mu \mathrm{M}$, and $6 \mu \mathrm{M})$ for 48 hours since studies have shown that $5 \mu \mathrm{M}$ SE treatment induces apoptosis in prostate cancer cells, but not in normal prostate cells. Cell cultures were examined under a Nikon inverted microscope and photographed with an attached Roper digital camera. MTS/PMS Cell Titer AQ Assay (Promega) was performed to determine cell viability. Apoptosis was evaluated using gel electrophoresis to detect DNA laddering. Western blotting was used to measure the levels of different forms of caspase-3.

Results: Direct microscopic examination revealed obvious cell death of EC cells after $\mathrm{SE}$ treatment. The cell viability assay showed that, after a slight increase of viable cells with $2 \mu \mathrm{M}$ SE treatment, cell viability decreased by $54 \%$ and $77 \%$ with $4 \mu \mathrm{M}$ and $6 \mu \mathrm{M}$ SE treatment, respectively. Apoptosis occurred with $2 \mu \mathrm{M}$ SE treatment and increased as the concentration of SE rose. The $17 \mathrm{kDa}$ subunit of caspase- 3 was not detectable without SE treatment, although it became detectable after treatment with $2 \mu \mathrm{M} \mathrm{SE}$, and the level increased 10-fold after $4 \mu \mathrm{M}$ SE treatment. The $20 \mathrm{kDa}$ peptide and the $11 \mathrm{kDa}$ subunit only increased slightly with $2 \mu \mathrm{M}$ or higher concentrations of SE treatment. The level of $32 \mathrm{kDa}$ precursor seemed unchanged after SE treatment

Conclusions: To our knowledge, this is the first study showing an anti-cancer effect of $\mathrm{SE}$ in EC, associated with induction of apoptosis. The concurrent increase of an active, $17 \mathrm{kDa}$ subunit, but not the other forms of caspase-3, suggests that the $17 \mathrm{kDa}$ subunit is more important in the caspase- 3 pathway of the SE-induced apoptosis in EC. 